American Journal of Infectious Diseases 6 (2): 34-39, 2010

ISSN 1553-6203

(C) 2010 Science Publications

\title{
A Study on Infections Caused By Metallo Beta Lactamase Producing Gram Negative Bacteria in Intensive Care Unit Patients
}

\author{
Debasrita Chakraborty, Saikat Basu and Satadal Das \\ Department of Microbiology and Serology, \\ Peerless Hospital and B.K. Roy Research Centre, \\ 76 Satyen Roy Road, Kolkata 700094, India
}

\begin{abstract}
Problem statement: Metallo Beta Lactamse (MBL) producing bacteria is gradually increasing throughout the globe. There is no report of MBL producing bacteria from the city of Kolkata so far although it is a very big metropolice city in India. Thus this study was aimed to investigate the impact of this highly virulent group of bacteria in this city. Approach: In this experiment we studied the prevalence, following standard methods of isolation and identification techniques of these bacteria from clinical materials and also studied some characteristics and clinical data in relation to MBL producing bacterial infections in this locality. Results: It was seen that a high prevalence of MBL producing bacteria was present in this city and there were many differences between MBL producing bacterial infection in comparison to the MBL non producing bacterial infection, particularly in relation to age distribution, sex predominance, mortality rate, hematological changes, nature of primary diseases in which infection occurred. There were also some electron microscopic morphological alterations in MBL positive bacterial isolates. Conclusion: This study confirmed significant occurrence of MBL producing bacterial infections in Kolkata showing distinct clinic microbial changes in this type of infection.
\end{abstract}

Key words: Metallo beta lactamase, gram negative bacteria, TEM study

\section{INTRODUCTION}

In India treatment of intensive care unit patients is becoming difficult due to upsurge of carbapenemases in Gram negative bacteria belonging to Enterobacteriaceae. Carbapenems, most specifically Imipenem and Meropenem have been considered as the most potent beta lactams against Multi Drug Resistant (MDR) Gram negative bacteria. But extensive and sometime unnecessary use of the carbapenems, poor sanitation and large population has facilitated the emergence of carbapenem resistant bacteria. Two types of carbapenem hydrolyzing enzymes are there, one is serine beta lactamase (having Serine at their active site) and other is Metallo Beta Lactamase (MBL), containing metal ion that works as a cofactor for enzyme's activity (Bush, 1999; 2001). Throughout the world bacteria like Serratia marcescens, Klebsiella pneumoniae, Citrobacter freundii, Pseudomonas aeruginosa, Pseudomonas putida, Acinetobacter spp. and Alcaligens xylosoxidans are generally noted as MBL producers. MBL can hydrolyze all metallo beta lactams except monobactams and are not inactivated by beta lactamase inhibitors like clavulanic acid, sulbactam and tazobactam (Daoud et al., 2008). The most useful and widely accepted classification of beta lactamase is Ambler (1980) classification that divided beta lactamases into four classes- A, B, C and D, where MBL is the class B beta lactamase. Bush (1989) further classified MBLs according to their functional properties into a separate group i.e., Group 3. This classification is based on the mechanism of action of the enzyme towards substrate, their sensitivity to EDTA and lack of inhibition by serine beta lactamase inhibitor. Five different types of MBLs whose prevalence are increasing rapidly are IMP, VIM, SPM, GIM and SIM (Luzzaroa et al., 2004; Walsh et al., 2005; Sadar et al., 2005), among them IMP and VIM are most predominant (Senda et al., 1996).

New subtypes of IMP and VIM are constantly being reported and recently 11 subtypes of VIM and 21 subtypes of IMP have also been studied (Lagatolla et al., 2004; Poirel et al., 2000). IMP-1 was first identified in $P$. aeruginosa and Acinetobacter spp. in Japan

Corresponding Author: Satadal Das, Department of Microbiology and Serology,

Peerless Hospital and B.K. Roy Research Centre, 76 Satyen Roy Road, Kolkata 700034, India

Tel: +91-09831092130, 033-2462 2394 Fax: 91(033) 24620766 
(Lauretti et al., 1999). Carbapenems are most potent beta lactams against beta lactamase producing bacteria but carbapenem resistance are increasing among Enterobacteriaceae, $P$. aeruginosa and in Acinetobacter spp. The common reasons for resistance may be due to the mutation of efflux pumps so that drug cannot penetrate through them, or hyper production of an AmpC type beta lactamase and other beta lactamases (Walsh et al., 2005). Metallo beta lactamase producing Gram negative bacteria often exhibit resistance to additional classes of drugs and behave as multi-drug resistant bacteria (Lee et al., 2005). This study was aimed to find out the characteristics and nature of MBL producing bacterial infection towards the patients of some Kolkata hospitals along with their structural alteration.

\section{MATERIALS AND METHODS}

Collection of Gram negative bacilli from ICCU and ITU: Over a 6 month period Gram negative bacterial species were isolated from various clinical specimens from ICCUs and ITUs of tertiary care hospitals in Kolkata. Usual laboratory methods were done to identify these bacterial strains as described elsewhere (Forbes et al., 2007). Some clinical associated data (age, sex, primary disease, mortality, associated predisposing diseases like diabetes.) of patients along with their consent were taken. Antibiogram of MBL producing and non producing bacterial strains were studied.

Study of MBL Production: Currently no standardized method for MBL detection has been proposed and despite PCR being highly reliable and accurate, for routine laboratory study some phenotypic methods were followed.

Combined disc diffusion method: Two $10 \mu \mathrm{g}$ Imipenem discs were placed on the Mueller Hinton (MH) media inoculated with test organism (maintaining $0.5 \mathrm{McF}$ arland standards). To one of the Imipenem discs, $0.5 \mathrm{M} 10 \mu \mathrm{L}$ EDTA solution was added. After $16 \mathrm{~h}$ of incubation at $35^{\circ} \mathrm{C}$, the zone of inhibition around Imipenem and Imipenem + EDTA discs were compared. If the zone of inhibition of Imipenem + EDTA discs compared to Imipenem alone is greater than $7 \mathrm{~mm}$, then the test organism can be considered as MBL producing. This procedure for MBL detection was done by Yong et al. (2002). The same test was done with Meropenem and Ceftazidime discs also with EDTA.

Double disc synergy test: Imipenem discs $(10 \mu \mathrm{g})$ was placed on the MH media inoculated with test organism and 10-25 mm apart a blank filter study disc was placed to which $0.5 \mathrm{M} 5 \mu \mathrm{L}$ EDTA solution was added, another Imipenem disc was placed on the far side. The zone of inhibition around Imipenem disc if expands towards EDTA disc, compared to the other Imipenem disc, placed on the far side, could be interpreted as a positive result (Lee et al., 2003). The same test was done with Meropenem disc and Ceftazidime disc with $2 \mu \mathrm{L}$ of concentrated 2-mercaptopropionic acid also.

Confirmation of MBL by E-test: It is the most sensitive test to detect MBL production. Bacterial suspension of $0.5 \mathrm{McFarland}$ was spread on $\mathrm{MH}$ agar medium by sterile swab stick. Then E-test strip which contains double sided seven dilution range of Imipenem (4-256 $\mu \mathrm{g} \mathrm{mL}^{-1}$ ) and seven dilution range of Imipenem $\left(164 \mu \mathrm{g} \mathrm{mL}^{-1}\right)$ with fixed concentration of EDTA, was placed on the surface of the medium and the plates were incubated at $35^{\circ} \mathrm{C}$ in air for $16-24 \mathrm{~h}$. After incubation the ratio of MIC values of Imipenem to Imipenem-EDTA was calculated. If it is greater than 8 , then the strain could be recorded as MBL producing strain. The extended zone between the junction of IMP and IMPEDTA is called as phantom zone. If phantom zone appears, then it was also be counted as positive result (Walsh et al., 2002).

Study of biochemical identification parameters of isolated microorganism: All routine tests like TSI (triple sugar iron), citrate, urease, oxidase, catalase, lactose, methyl red and indole were done to verify the bacterial strains according to standard procedures (Forbes et al., 2007).

Antimicrobial susceptibility study of isolated microorganism with determination of MIC was done according to CLSI guidelines.

Transmission Electron Microscopic (TEM) study of isolated MBL positive bacteria: One strain of MBL producing Pseudomonas aeruginosa were selected to see any structural difference comparing to international strain. In this study an international strain of Pseudomonas-Pseudomonas aeruginosa ATCC27853 and an MBL producing Pseudomonas aeruginosa (PH7506) were tested. Their structural differences were noted in TEM, (FEI and TECHNAI G ${ }^{2}$ ). The cells were prepared for TEM and then kept in $70 \%$ alcohol. Prior to treatment it was dehydrated in absolute alcohol. It was kept for 1.5-2 min after the sample was placed on the grid. Then excess amount of the sample was absorbed by touching blotting paper strip on the one side of the carbon coated copper grid and was kept for $45 \mathrm{sec}$ to $1 \mathrm{~min}$. Similarly excess stain was removed by blotting paper and then the cells were seen under microscope. 


\section{RESULTS}

Differences in studies of MBL positivity: Both combined disc diffusion method and double disc synergy test showed very clear result for detecting MBL production. If we compare between the two tests then it has been seen that both of the methods more or less have same accuracy but one method can give most accurate result to one type of strain while the other method may be appropriate for some other strains. For confirmation of MBL production in laboratory both tests should be done simultaneously along with the Etest. E-tests give most accurate result having sensitivity and specificity above $90 \%$. But there is no perfect detection method in laboratory that can detect all MBLs other than PCR.

General prevalence of MBL positive bacteria in Kolkata: Prevalence of MBL producing bacteria was studied and it was seen that $41.2 \%$ bacteria were MBL positive among all isolates. Out of total Gram negative strains were isolated, $40.20 \%$ were Pseudomonas spp. 31 and $29 \%$ were Klebsiella spp. and E. coli respectively. It was found that out of total isolated Pseudomonas spp. $51.28 \%$ were MBL positive, where as Klebsiella it was $36.6 \%$ and for E. coli the MBL positivity was $28.57 \%$. In case of MBL positive Pseudomonas spp. $45 \%$ strains were Multi Drug Resistant (MDR), these were resistant to gatifloxacin, netilmicin, third generation cephalosporins and carbapenems.

Findings of TEM study: MBL positive Pseudomonas aeruginosa were found on an average slightly longer and thicker (average size $1.81 \times 0.61 \mu \mathrm{m}$ ) than the international ATCC 27853 Pseudomonas aeruginosa (average size $1.73 \times 0.59 \mu \mathrm{m}$ ). They contain very small scanty vacuoles with uniform size (2-4vacuoles, average size $0.034 \mu \mathrm{m})$ than those in the international ATCC 27853 Pseudomonas aeruginosa (5-8vacuoles, irregular size, range $0.07-0.41 \mu \mathrm{m})$. MBL positive Pseudomonas aeruginosa also contains capsule (average thickness $0.057 \mu \mathrm{m}$ ) with many distinct flagella.

Difference between MBL positive and MBL negative bacteria in relation to the patients suffering from infection on the basis of clinical data: From hospital patients various clinical parameters were collected along with their consent.

Age distribution: Age distribution of patients having infection with (MBL) positive organisms were mainly belong to 61-80 years age group. However age distribution of patients having infection with MBL negative organisms were mainly in between 51-70 years of age (Fig. 1).

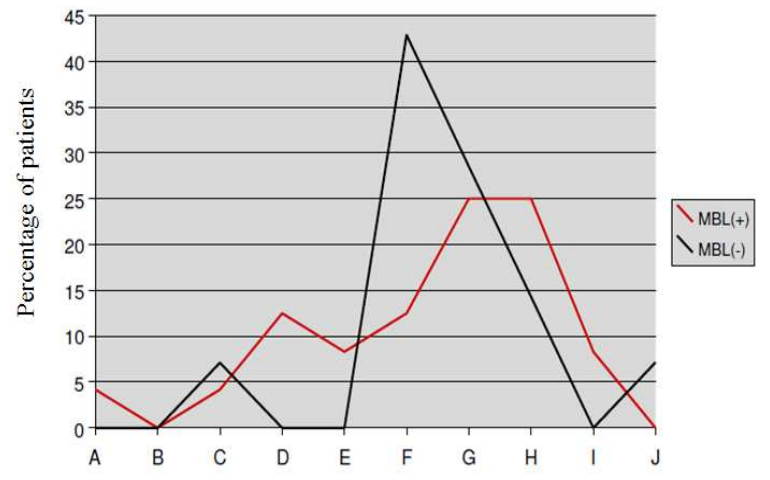

Fig. 1: AGE distribution of patients having infection with MBL (+) and MBL (-) bacteria. Age in years; A: 0-10; B:11-20; C: 21-30; D: 31-40; E: 41-50; F: 51-60; G: 61-70; H: 71-80; I: 81-90; J: $91-100$.

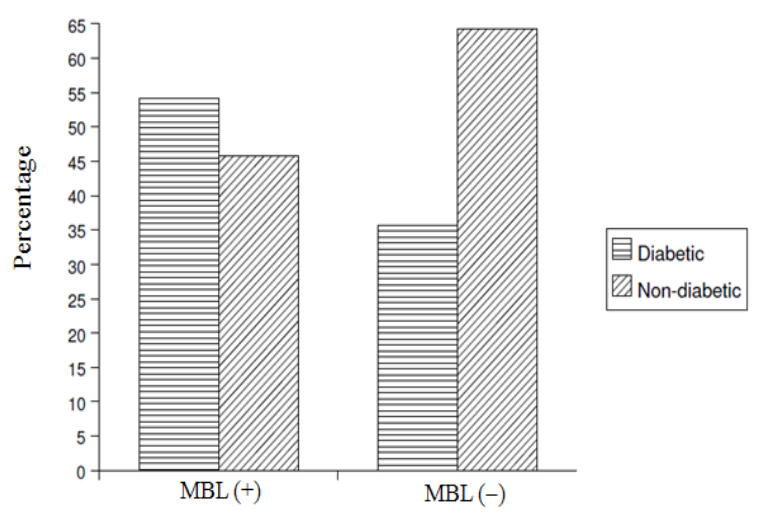

Fig. 2: Mortality rate in diabetic and non-diabatic patients in relation to MBL positivity

Sex predominance: The analysis of sex distribution of patients with MBL positive microbial infections revealed male preponderance $(66.8 \%)$ and only $33.2 \%$ patients with similar infections were females. If we compare this with patients with MBL negative microbial infections, then it is highly significant to note that there is no sex predominance in this group, male and females were both showing $50 \%$ of occurrence.

Mortality: Among patients infected with MBL positive Gram negative bacteria, the death percentage was $54.6 \%$ while in case of patients infected with MBL negative Gram negative bacteria the percentage was $21.42 \%$. About $54.16 \%$ patients infected with MBL producing bacteria were diabetic while only $35.7 \%$ patients having non MBL bacterial infections were reported as diabetic. It was seen that mortality rate is high in diabetic patients (Fig. 2). 
Antibiogram of MBL producing and nonproducing Gram negative bacteria: $\mathrm{MBL}$ producing bacteria were mainly isolated from ICCU and ITU and we screened only few selected group of antibiotics which are commonly used in critical care units in India. In this study we screened third generation Cephalosporins; Fluoroquinolones; Aminoglycosides; Carbapenems (imipenem, meropenem); Aztreonam and Colistin. In general it was found that MBL positive microorganisms were highly resistant to all these antibiotics in comparison to MBL negative microorganisms (Fig. 3). MBL positive bacteria showed almost $100 \%$ resistance to third generation Cephalosporins (cephotaxime, ceftazidime, ceftriaxone) while for MBL negative organism it is between (23-61\%). Even if we consider the newer antibiotics like Imipenem, Meropenem, high resistance against these antibiotics for MBL positive bacteria were also noticed. Here $14 \% \mathrm{MBL}$ negative bacteria were resistant to Imipenem, while in case of MBL positive bacteria $78.25 \%$ resistance was found. If we look at the Quinolone group of antibiotics (levofloxacin, ofloxacin, ciprofloxacin, gatifloxacin), it was seen that MBL positive E. coli showed maximum resistance $(70 \%)$ against ofloxacin while only $40 \% \mathrm{MBL}$ non producing $E$. coli is resistant to this. MBL positive Pseudomonas spp. and E. coli showed high resistance $(74-78 \%)$ to ciprofloxacin compared to their negative counterparts (Fig. 3).

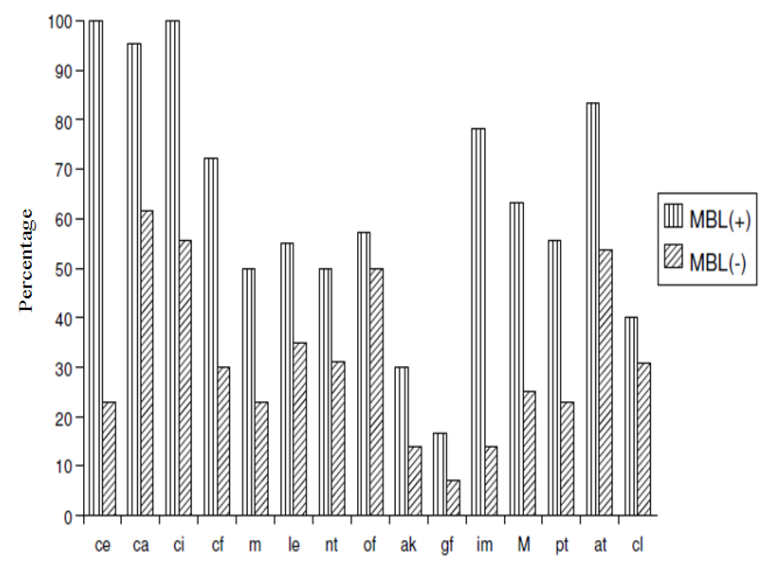

Fig. 3: Antibiotic resistance of MBL (+) and MBL (-) organisms; ce: Cefataxime; ca: Ceftazidime; ci: Ceftriaxone; cf: Ciprofloxacin; m: magnex; le: Levofloxacin; nt: Netilmicin; of: Ofloxacin; ak: Amikacin; gf: Gatifloxacin; im: Imipenem; M: meropenem; Pt: Piperacillin + tazobactam; at: Aztreonam; cl: Colistin
Routine haematological parameters in infections with MBL positive organisms: In this study the mean Total Count (TC) in patients with MBL producing bacterial infection was not significantly altered, when compared to mean TC in similar patients with MBL negative bacterial infection. The mean TC with Standard Deviation (SD) and Standard Error Mean (SEM) in patients with MBL positive bacterial infection were $17919.54 \pm 20414.5 \pm 4359.6 \mathrm{~mm}^{-3}$ of blood which was almost similar to mean TC with SD and SEM in MBL negative infection which were $14111.36 \pm 7545.95 \pm 1608.80 \mathrm{~mm}^{-3}$ of blood (t-value $=$ \pm 0.9179 , the two tailed p-value is 0.3691 , by conventional criteria, this difference is considered to be not statistically significant).

Similarly if we compare mean neutrophil percentage in these two groups then there was also no statistically significant difference in two groups. Mean neutrophil percentage with SD and SEM in MBL positive bacterial infection was $79.14 \pm 18.55 \pm 3.95 \%$ which was almost similar to MBL negative bacterial infection group with mean neutrophil percentage with SD and SEM 84.41 $\pm 7.77 \pm 1.66 \%$ (t-value $= \pm 1.18$, the two tailed $\mathrm{p}$ value was 0.2484 , this difference was also considered to be not statistically significant).

Mean ESR with SD and SEM in MBL positive bacterial infection were $88.72 \pm 30.21 \pm 6.44 \mathrm{~mm} \mathrm{~h}^{-1}$ and in MBL negative bacterial infection it was $36 \pm 32.63 \pm 6.96 \mathrm{~mm} \mathrm{~h}^{-1}$ (t-value was \pm 6.513 ; $\mathrm{p}$-value is less than 0.0001 , this difference was considered to be extremely statistically significant) (Table 1 and 2).

Primary disease: Among patients, infected with the MBL producing bacteria, 31\% were affected by the Chronic Obstructive Pulmonary Disease (COPD). Hepatic failure, cancer, cardiac disease was also observed in $10.5 \%$ cases each. $21 \%$ cases were suffering from pneumonia and $15.8 \%$ cases were patients of renal failure. It was been seen that the MBL producing bacteria can cause multi organ failure in hospitalized patients, in hospitals of this locality $52.8 \%$ cases of total MBL positive bacteria infected patients multi organ failure had been observed.

Table 1: Mean, SD and SEM values of WBC counts and ESR for patients with MBL producing bacterial infections

\begin{tabular}{lrrr}
\hline & \multicolumn{1}{l}{ Mean } & \multicolumn{1}{l}{ SD } & \multicolumn{1}{l}{ SEM } \\
\hline Total WBC count $\left(\mathrm{m}^{-3}\right.$ of blood) & 17919.55 & 20448.57 & 4359.65 \\
Neutrophill $(\%)$ & 79.14 & 18.55 & 3.95 \\
ESR $\left(\mathrm{mm} \mathrm{h}^{-1}\right)$ & 88.73 & 30.21 & 6.44 \\
\hline
\end{tabular}

Table 2: Mean, SD and SEM values for WBC counts and ESR in patients with non MBL producing bacterial infections

\begin{tabular}{lrrr}
\multicolumn{4}{c}{ patients with non MBL producing bacterial infections } \\
\hline & Mean & \multicolumn{1}{c}{ SD } & \multicolumn{1}{c}{ SEM } \\
\hline Total count $\left(\mathrm{m}^{-3}\right.$ of blood) & 14111.36 & 7545.95 & 1608.80 \\
Neutrophil $(\%)$ & 84.41 & 7.77 & 1.66 \\
ESR $\left(\mathrm{mm} \mathrm{h}^{-1}\right)$ & 36.00 & 32.63 & 6.96 \\
\hline SD: Standard Deviation; SEM. Standard Error of Mean
\end{tabular}


Diabetes mellitus and MBL positive Infections: Patients suffering from Diabetes mellitus are commonly known to suffer from various infectious diseases much more than the general population. In this study $54.16 \%$ subjects belonging to MBL positive group, were suffering from diabetes mellitus with an average blood glucose level of $250 \mathrm{mg} \mathrm{dL}^{-1}$. In patient groups infected with MBL negative bacteria only $35.7 \%$ were suffering from Diabetes mellitus. This clearly indicates that Diabetes mellitus is an important predisposing factor of infections caused by MBL positive Gram negative organisms.

\section{DISCUSSION}

This study shows the prevalence and other characteristics of metallo beta lactamase producing Gram negative bacteria in Kolkata hospitals for the first time. Resistance to carbapenems in Pseudomonas spp. causing nosocomical infection has been increasing in recent years in Kolkata hospitals indicated that those MBLs have started to represent a countrywide problem. From TEM study it was seen that MBL producers have some differences on their cell size and structures with MBL non producers. MBL producing Pseudomonas spp. contained capsules and prominent flagella that may indicate their mode of resistance.

In reference to of age distribution of the patients it was observed that MBL positive organisms were mainly isolated from infections in old age group of patients. The age distribution in the patients followed more or less a bell shaped pattern and there is no multiphase distribution. This may indicate that if at all there is a human genetic influence determining the infection of MBL positive organism, it should belong to a single genetic MBL locus. Affection of high age group by MBL positive organisms may be due to their general debilitating condition, due to senility and senility associated cellular damage and diseases.

While discussing about sex distribution the result indicates that male individuals are very much prone to infections with MBL positive organisms. This may be due to presence of some protective factors in females or presence of some aggressive factors in males allowing easy infection by these organisms.

Mortality rate is higher in case of patients infected with MBL producing bacteria than that with MBL negative bacteria. MBL producing bacteria cause more serious type of disease that is non treatable or may lead to death. If we consider the rate of mortality, then it was found that among patients suffering from diabetes mellitus having MBL positive microbial infection, were prone to higher degree of mortality $(58.3 \%)$ than in patients who are not suffering from diabetes mellitus (42\%). Preventive care should be initiated in critical care units of all hospitals to prevent MBL positive infections.

Antibiotic resistance pattern among MBL producing and nonproducing bacteria indicates that MBL positive bacteria are much more resistant to antibiotics due to formation of different types of antibiotic inactivating enzymes. Resistance pattern against a broad group of antibiotics may indicate formation of many such enzymes by MBL positive group of bacteria. Thus in this way MBL producing bacteria mostly show multi drug resistance.

Although there were no significant changes in TLC and neutrophil percentage in between MBL positive and MBL negative bacterial infection, however, if we consider ESR level then there was a highly significant change in between MBL positive and MBL negative bacterial infection.

Further investigation is required to have a better understanding of the epidemiology and genetic background of MBL producing bacteria. Longer hospital stay, prolong use of antibiotics (especially carbapenems) are possibly the main risk factors for the emergence of the Gram negative MBL producers (Livermore and Woodford, 2000; Nordman and Poirel, 2002). The uncontrolled spread of MBL producers in hospitals may hamper the treatment procedures with increased morbidity and mortality. Regular screening system should be established to arrest the spread of the MBL producers and effective infection control programs in hospitals should be developed and maintained thoroughly.

\section{CONCLUSION}

There is a significant prevalence of MBL producing Gram negative bacteria in patients of intensive care units of Kolkata, which mainly affects male patients belonging to the old age group. Mortality of the patients infected with MBL producing bacteria is also very high mainly due to multi organ failure. There are also some distinct morphological feature in MBL producing bacteria.

\section{ACKNOWLEDGMENT}

We are most grateful to the managing director of Peerless Hospital and B.K. Roy Research Centre for giving us funds and clinical data.

\section{REFERENCES}

Ambler, R.P., 1980. The structure of $\beta$-lactamases. Phil. Trans. R. Soc. Lond. B. Biol. Sci., 289: 321-331. PMID: 6109327 
Bush, K., 1989. Classification of $\beta$-lactamases-group2c, group-2d, group-2e, group-3 and group-4. Antimicrob. Agents Chemother. 33: 271-276. PMID: 2658781

Bush, K., 1999. $\beta$-lactamases of increasing clinical importance. Curr. Pharm. Des., 5: 839-845. PMID: 10539991

Bush, K., 2001. New $\beta$-lactamases in gramnegative bacteria: Diversity and impact on the selection of antimicrobial therapy. Clin. Infect. Dis., 32: 1085-1089. DOI: 10.1128/AAC.50.1.388390.2006

Daoud, Z., E. Hobeika and A. Choucair, 2008. Isolation of the first metallo-beta-lactamase producing Klebsiella pneumoniae in Lebanon. Rev. Esp. Quimioter, 21: 123-126. PMID: 18509771

Forbes, B.A., D.F. Sahm and A.S. Weissfeld, 2007. Baily and Scott's Diagnostic Microbiology. 12th Edn., Elsevier, Mosby, pp: 323-350.

Lagatolla, C., E.A. Tonin, C. MontiBragadin, L. Dolzani and F. Gombac et al., 2004. Endemic carbapenem-resistant Pseudomonas aeruginosa with acquired metallo- $\beta$ lactamase determinants in European hospital. Emerg. Infect. Dis., 10: 535-538. PMID: 15109432

Lauretti, L., M.L. Riccio, A. Mazzariol G. Cornaglia and R. Amicosant Fontana et al., 1999. Cloning and characterization of blaVIM, a new integronborne metallo- $\beta$-lactamase gene from a Pseudomonas aeruginosa clinical isolate. Antimicrob. Agents Chemother., 43: 1584-1590. PMCID: PMC89328

Lee, K., Y.S. Lim, D. Yong, J.H. Yum and Y. Chong, 2003. Evaluation of the Hodge test and the imipenem-EDTA double disk synergy test for differentiation of metallo- $\beta$-lactamases producing clinical isolates of Pseudomonas spp. and Acinetobacter spp. J. Clin. Microbiol., 41: 4623-4629. DOI: 10.1128/JCM.41.10.4623-4629.2003

Lee, K., J.H. Yum, D. Yong, H.M. Lee and H.D. Kim et al., 2005. Novel acquired metallobeta-lactamase gene, blaSIM-1, in a class 1 integron from Acinetobacter baumannii clinical isolates from Korea. Antimicrob. Agents Chemother., 49: 4485-4491. DOI: 10.1128/AAC.49.11.4485-4491.2005

Livermore, D.M. and N. Woodford, 2000. Carbapenemases: A problem in waiting? Curr. Opin. Microbiol., 3: 489-495. PMID: 11050448
Luzzaroa, F., A. Endimiania, J.D. Docquierb, C. Mugnaioli and G. Amicosante et al., 2004. Prevalence and characterization of metallo-beta-lactamases in clinical isolates of Pseudomonas aeruginosa. Diagn. Microbiol. Infect Dis., 48: 131-135. DOI: 10.1016/j.diagmicrobio.2003.09.005

Nordman, P. and L. Poirel, 2002. Emerging carbapenemases in gram-negative aerobes. Clin. Microbiol. Infect., 8: 321-331. PMID: 12084099

Poirel, L., T. Naas, D. Nicolas, L. Collet and S. Bellais et al., 2000. Characterization of VIM-2, a carbapenem-hydrolyzing metallo- $\beta$-lactamase and its plasmidand integron borne gene from a Pseudomonas eruginosa clinical isolate in France. Antimicrob. Agents Chemother., 44: 891-897. PMID: 17927473

Sadar, H.S., A.O. Resis, S. Silbert and A.C. Gales, 2005. IMPs, VIMs and SPMs: The diversity of metallo-beta-lactamase produced by carbapenemresistant Pseudomonas aeruginosa in a Brazilian hospital. Clin. Microbiol. Infect., 11: 73-76. PMID: 15649310

Senda, K., Y. Arakawa, K. Nakashima, H. Ito and S. Ichiyama et al., 1996. Multifocal outbreaks of metallo-beta-lactamaseproducing Pseudomonas aeruginosa resistant to broad-spectrum beta lactams, including carbapenems. Antimicrob. Agents Chemother., 40: 349-353. PMID: 8834878

Walsh, T.R., A. Bolmstrom, A. Qwarnstrom and A. Gales, 2002. Evaluation of a new $E$ test for detecting metallo- $\beta$-lacatamases in routine clinical testing. J. Clin. Microbiol., 40: 2755-2759. DOI: 10.1128/JCM.40.8.27552759.2002

Walsh, T.R., M.A., Toleman, L. Poirel and P. Nordmann, 2005. Metallo-beta-lactamse: The quiet before the storm? Clin. Microbiol. Rev., 18: 306-325. DOI: 10.1128/CMR.18.2.306-325.2005

Yong, D., K. Lee, J.H. Yum, H.B. Shin and G.M. Rossolini et al., 2002. Imipenem-EDTA disk method for differentiation of metallo- $\beta$-lactamases producing clinical isolates of Pseudomonas spp. and Acinetobacter spp. J. Clin. Microbiol., 40: 3798-3801. DOI: 10.1128/JCM.40.10.37983801.2002 